\title{
Extended multi-drug maternal therapy for refractory supraventricular tachycardia in premature hydrops fetalis
}

\author{
${ }^{1}$ Women's Wellness and Research Centre, Hamad Medical Corporation, Department of Obstetrics and Cyne- \\ cology, PO Box 3050, Doha, Qatar, E-mail: fathim999@gmail.com. https://orcid.org/0000-0001-6903-5445, \\ https://orcid.org/0000-0001-6058-3437. \\ ${ }^{2}$ Department of Pediatrics, Hamad Medical Corporation, Doha, Qatar
}

\begin{abstract}
:
Background: Fetal supraventricular tachycardia (SVT) is a reversible cause of fetal hydrops, with better outcomes if detected early and reverted in utero. Modern imaging allows accurate diagnosis and maternal antiarrhythmic therapy can be practiced ensuring in utero cardioversion.

Case presentation: We present a case of preterm fetal hydrops due to SVT successfully reverted in utero by multidrug maternal therapy. The mother presented to our tertiary care hospital at 32 weeks' gestation with a fetal heart rate of 229 beats/min with evidence of mild fetal pericardial effusion and ascites. Under strict maternal monitoring, the treatment was started with parenteral digoxin. Flecainide, sotalol and amiodarone were introduced sequentially based on the response until cardioversion was achieved at 35 weeks with no maternal side effects. She labored spontaneously and delivered a $2.6 \mathrm{~kg}$ baby boy 4 days after the successful cardioversion. The baby was discharged home on day 7 on oral amiodarone with outpatient follow-up. Digoxin monotherapy failed in our case, possibly due to evidence of hydrops and a sequential multi-drug therapy was required for an extended duration of up to 2 weeks to achieve cardioversion successfully.

Conclusions: Our report suggests that multi-drug therapy is more successful in cases of SVT with fetal hydrops, titrated according to fetal response and maternal tolerance, although a prolonged period of therapy might be required to achieve the desired clinical effect.
\end{abstract}

Keywords: Amiodarone, digoxin, fetal SVT, flecainide, in utero cardioversion, sotalol

DOI: $10.1515 / \mathrm{crpm}-2018-0034$

Received: June 9, 2018; Accepted: November 27, 2018

\section{Introduction}

Fetal tachycardia occurs in $0.5 \%$ of all pregnancies, of which the most common is supraventricular tachycardia (SVT) (90\%). If left untreated, it leads to cardiac failure, hydrops and fetal death. Modern imaging like three-dimensional/four-dimensional (3D/4D), Doppler ultrasonography and M-mode echocardiography allows accurate diagnosis and maternal administration of antiarrhythmic drugs is recommended to achieve in utero cardioversion. In non-hydropic fetuses, this decreases the progression to fetal hydrops and maintaining sinus rhythm in utero reduces the need for premature delivery. However, the management is more challenging when hydrops has already set in, possibly due to the poor transplacental transfer of medications across a hydropic placenta, and poor fetal circulation.

Many case series report digoxin outpatient monotherapies as well tolerated by the mother and successful in cardioversion of non-hydropic fetuses. However, there is no standardized regime for the management of premature hydropic fetuses due to the lack of randomized trials, and various case reports suggest inpatient multiple drug maternal therapy with intensive maternal and fetal monitoring.

We present a case of premature fetal SVT with hydrops detected at 32 weeks, successfully managed by maternal multidrug therapy by a multidisciplinary team including pediatric cardiology, adult cardiology and the fetal-maternal unit. This case required a trial of digoxin monotherapy followed by combination with flecainide, sotatol and amiodarone. The good maternal tolerance permitted us to continue the therapy until cardioversion was achieved on day 14 of admission that resulted in the birth of a baby at near-term gestation with sinus rhythm and resolved hydrops. 


\section{Case presentation}

The mother, a 31-year-old gravida 3 para 1, presented for a booking visit at our tertiary care hospital at 32 weeks +5 days' gestation by her dates, and was diagnosed with fetal SVT with evidence of cardiac failure on her first ultrasound scan. She previously delivered a healthy baby boy of $2.67 \mathrm{~kg}$, vaginally at 36 weeks following preterm premature rupture of membranes and spontaneous labor. An uncomplicated first trimester miscarriage preceded the current pregnancy. There was no significant past surgical or medical history. She gave no family history of cardiac disease or thyroid disease, and no history of smoking, or excessive consumption of caffeine, alcohol or any illegal drugs. Her blood group was AB positive with a negative antibody screen. Her pregnancy had been uneventful, and she appreciated fetal movements. Her physical examination was unremarkable with stable vital signs and a body mass index of 25.

Real-time ultrasound evaluation of the fetus revealed an intrauterine, singleton pregnancy with all fetal parameters appropriate for gestational age, in cephalic presentation. There was adequate amniotic fluid volume and estimated fetal weight was $2108 \mathrm{~g}$. The placenta was normal with a three-vessel umbilical cord and central insertion. The fetal heart rate (FHR) was found to be $229 \mathrm{bpm}$, and there was evidence of mild pericardial effusion with ascites. The umbilical artery and middle cerebral artery Dopplers were normal with no evidence of fetal anemia. The patient was admitted to the high dependency unit to administer steroids for fetal lung maturity and to begin fetal cardioversion by administration of anti-arrhythmic drugs.

A multidisciplinary team, including the obstetrics and fetal-maternal team, pediatric cardiologist, adult cardiologist, and a neonatologist, was involved in the management. A maternal electrocardiogram (ECG) done on admission was normal. After ensuring a normal liver and kidney function tests with normal magnesium and calcium levels, she was started on intravenous digoxin, $0.5 \mathrm{mg}$, followed by $0.25 \mathrm{mg} 12$ hourly for three doses, with serum digoxin levels checked before each dose to maintain a therapeutic level of 1.5-2.5 nmol/L. As the SVT was persistent and the serum digoxin levels were suboptimal, $0.5 \mathrm{mg}$ intravenous (i.v.) digoxin was given for three more doses 8 hourly.

An M-mode echo done on day 5 showed impaired cardiac contractility due to dysrhythmia, SVT with 1:1 conduction, FHR of $235 \mathrm{bpm}$, and no other cardiac anomalies. There was minimal pericardial effusion but otherwise no fetal ascites. After 3 days of i.v. digoxin, she was shifted to oral digoxin $0.25 \mathrm{mg}$ twice daily along with oral flecainide 100-150 mg given 2-3 times per day for 4 days. The serum digoxin levels were in the optimal range, but there was no response even with the second medication. Flecainide was replaced with sotalol 160 $\mathrm{mg}$ twice daily for the next 4 days and on the fifth day amiodarone $800 \mathrm{mg} 3$ times a day was added. Fetal cardioversion was achieved on day 14 of admission with a baseline fetal heart rate of $120 \mathrm{bpm}$, with occasional bouts of tachycardia subsiding spontaneously (Table 1). The maternal heart rate showed a gradual drop below $60 \mathrm{bpm}$ on three occasions. The medications were held during these episodes allowing the mother to recover before resuming. No other side effects of the medications were noted, and the patient tolerated the medications well. The mother was monitored for side effects by continuous pulse and blood pressure charting, daily ECGs, weekly kidney liver function test and electrolyte balance, and digoxin levels before and after each dose of medications. The response in fetal heart rate according to the medications sequentially added, and the changes in maternal digoxin levels and maternal heart rate over the treatment days are as plotted in Figure 1.

Table 1: Medication administration schedule.

\begin{tabular}{|c|c|c|c|c|c|c|c|c|}
\hline \multirow{3}{*}{$\begin{array}{l}\text { GA } \\
\begin{array}{l}32 \text { weeks }+6 \\
\text { days }\end{array}\end{array}$} & \multicolumn{2}{|c|}{ Day of admission } & \multirow{2}{*}{$\begin{array}{r}\begin{array}{r}\text { Digoxin } \\
\text { level, } \\
\text { nmol/L }\end{array} \\
0\end{array}$} & \multirow{2}{*}{$\begin{array}{l}\text { Medication } \\
\\
\text { Digoxin IV } 500 \\
\mu \mathrm{g} / 50 \mathrm{~mL} \text { NS15 min }\end{array}$} & \multicolumn{2}{|c|}{$\begin{array}{r}\text { Maternal vitals: } \\
\text { pulse, bpm } \\
\text { BP, mm Hg }\end{array}$} & \multirow{2}{*}{$\begin{array}{l}\text { Maternal } \\
\text { ECG } \\
\text { NAD }\end{array}$} & \multirow{2}{*}{$\begin{array}{r}\text { FHR } \\
220\end{array}$} \\
\hline & Day 2 & $5 \mathrm{am}$ & & & 69 & $110 / 60$ & & \\
\hline & & $5 \mathrm{pm}$ & 0.85 & $\begin{array}{l}\text { Digoxin IV } 250 \\
\mu \mathrm{g} / 50 \mathrm{~mL} \text { NS - } 15 \\
\mathrm{~min}\end{array}$ & 74 & $110 / 70$ & & \\
\hline \multirow[t]{2}{*}{33 weeks } & Day 3 & $5 \mathrm{am}$ & 0.66 & $\begin{array}{l}\text { Digoxin IV } 250 \\
\mu \mathrm{g} / 50 \mathrm{~mL} \text { NS }-15 \\
\text { min }\end{array}$ & 80 & $100 / 50$ & NAD & 250 \\
\hline & & $5 \mathrm{pm}$ & 1.12 & $\begin{array}{l}\text { Digoxin IV } 250 \\
\mu \mathrm{g} / 50 \mathrm{~mL} \text { NS - } 15 \\
\mathrm{~min}\end{array}$ & 82 & & & \\
\hline $\begin{array}{l}33 \text { weeks }+1 \\
\text { day }\end{array}$ & Day 4 & $2 \mathrm{pm}$ & 1.21 & $\begin{array}{l}\text { Digoxin IV } 500 \\
\mu \mathrm{g} / 50 \mathrm{~mL} \text { NS }-15 \\
\text { min }\end{array}$ & 87 & $110 / 70$ & NAD & 235 \\
\hline
\end{tabular}




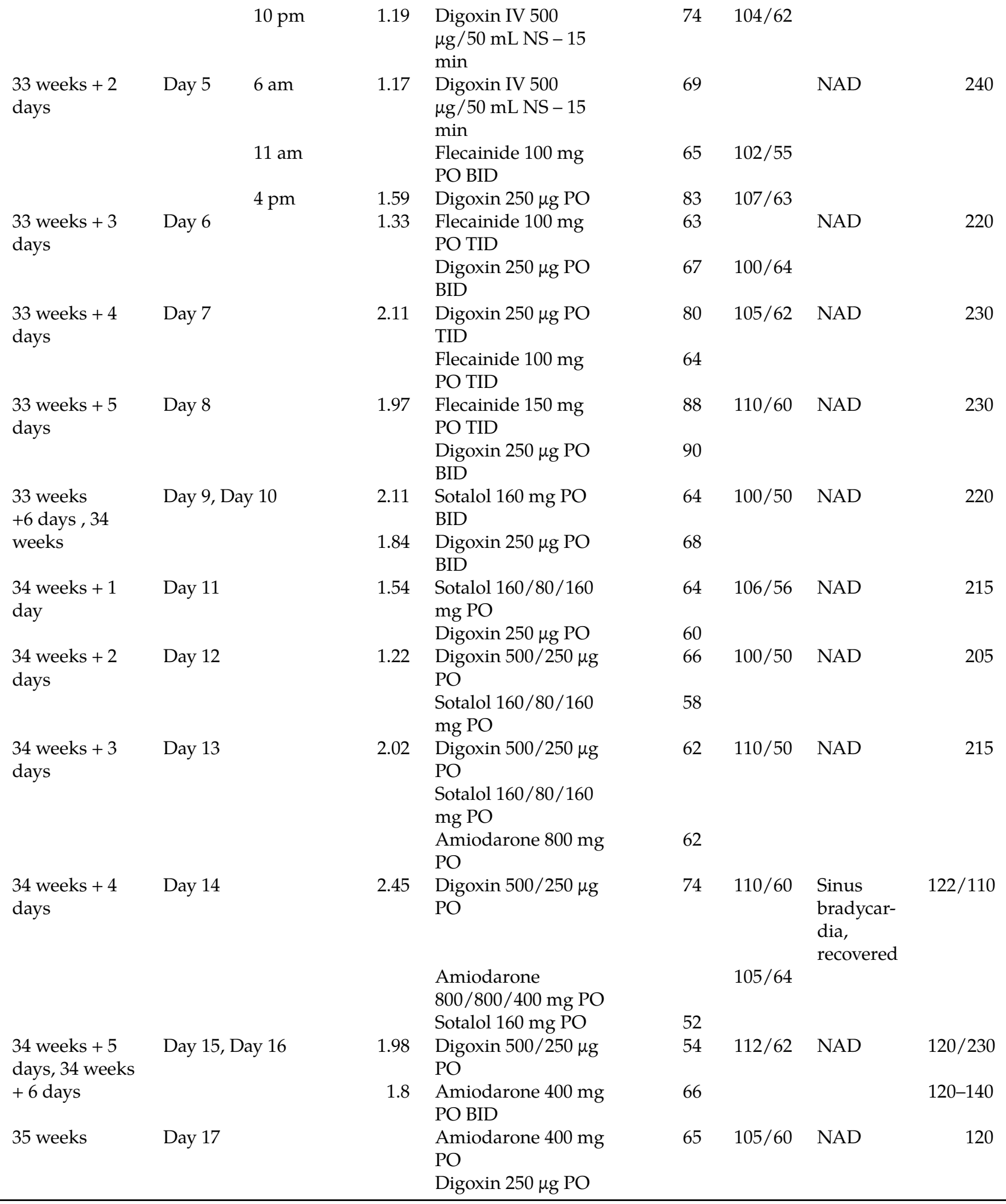

GA, gestational age; nmol/L, nanomoles per liter; bpm, beats per minute; ECG, electrocardiogram; FHR, fetal heart rate; mg, milligrams; $\mu \mathrm{g}$, micrograms; mL, milliliters; NS, normal saline; IV, intravenous; PO, per oral; OD, once daily; BID, twice daily; TID, thrice daily; NAD, no abnormality detected.

On day 17 of admission, the FHR baseline was 120-130 bpm with no episode of tachycardia for $24 \mathrm{~h}$. The patient went into spontaneous labor shortly afterwards and had an uncomplicated vaginal delivery at 35 weeks of gestation, giving birth to a $2.66 \mathrm{~kg}$ baby boy. All medications were stopped on delivery, and the mother was discharged after 3 days in stable condition.

Postnatally, the baby had two episodes of SVT treated with adenosine and was placed on amiodarone. Echocardiography showed a small patent foramen ovale with mild tricuspid regurgitation, normal systolic function and a thin rim of pericardial effusion. The hemoglobin was 16.5 at birth, with normal thyroid function 
tests. He was discharged on day 7 on amiodarone $12 \mathrm{mg}$ once daily after no episode of SVT noted for 5 days, with an outpatient follow-up with the pediatric cardiologist.

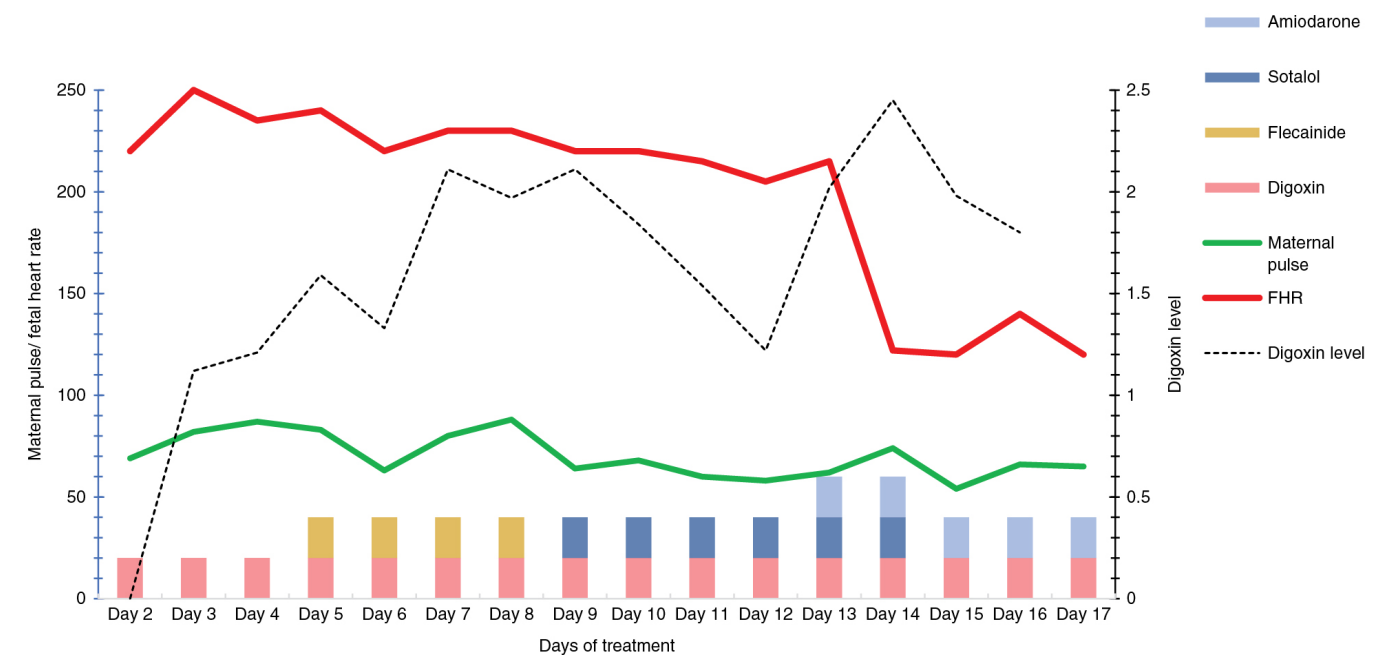

Figure 1: Changes in maternal pulse and fetal heart rate noted over the days of treatment.

On further follow-up, the infant required amiodarone and propranolol for up to 6 months of age after which the medications were tapered gradually and finally stopped at 1 year of age, as the frequency of the episodes decreased and he remained symptom free. The follow-up echocardiography done at 8 months of age showed no residual structural anomalies and an ejection fraction of $71 \%$. The 2 year old is currently fit and healthy, completely weaned off cardiac medications with no long-term consequences.

\section{Discussion}

SVT is characterized by a regular rate that is typically between 220 and $260 \mathrm{bpm}$, and when long standing, it results in ventricular dysfunction and fetal hydrops, which can quickly result in fetal death [1]. The etiology can include maternal factors like smoking, excess caffeine intake, illicit drug use, endocrine disorders or fetal factors like fetal cardiac anomalies or other congenital anomalies like diaphragmatic hernia. However, in many instances, like in our case, the etiology is unknown. The delivery of a premature hydropic baby with SVT is certainly not desirable due to the associated neonatal mortality and long-term neurological effects. The possibility of in utero cardioversion using anti-arrhythmic medications in cases where structural anomalies have been ruled out, reduces the need for preterm delivery and subsequent neonatal management of tachyarrhythmia. These medications have a good safety profile with good maternal tolerance even at the high doses required to achieve therapeutic ranges in pregnancy. Treatment is considered based on the rate and persistence of tachycardia, the gestational age at detection and the presence of hydrops, with preference given to persistent rate of more than $220 \mathrm{bpm}$ in a hydrops fetus of less than 34 weeks' gestation [2], [3], [4]. This practice is accepted worldwide especially in hydropic premature fetuses as this reduces the short- and long-term morbidity and mortality associated with premature delivery.

The most commonly used first line medication was digoxin, initiated parenterally followed by oral maintenance with outpatient follow-up, that achieved a good success rate in non-hydropic fetuses. However, there are many case series that report the decreased efficacy of monotherapy with persistent tachycardia more than $210 \mathrm{bpm}$ and hydrops [5]. Systematic reviews and meta-analyses found that flecainide was more successful in terminating SVT in comparison to digoxin in hydropic fetuses and could be a more appropriate first line drug [6], [7]. Various reports show the use of sotalol and amiodarone to terminate persistent SVT with good maternal safety profile [8], [9]. However, there is no clear consensus as to the medication schedule that can be universally used due to the lack of randomized studies.

In our case, a multi-drug therapy of digoxin, sotalol and amiodarone was administered under strict maternal monitoring, and the dosage titrated based on maternal tolerance and fetal response, till cardioversion took place on day 14 of admission. Although the SVT was persistent; serial ultrasound scans revealed no worsening of the hydrops. This permitted us to extend the duration of the therapy until the aim of delivering a baby maintaining sinus rhythm, without the added morbidity of prematurity was achieved. At delivery, the hydrops was found to have reversed completely, and the baby was discharged home on oral antiarrhythmic agents. Despite the extended therapy with multiple agents, the healthy mother tolerated the medications well and no side effects 
were encountered. She was admitted to a high dependency unit under close monitoring throughout admission. Our case shows that extended duration of therapy might be required in cases of hydrops with persistent SVT, which can be attained safely provided the mother is complaint and motivated, and the fetal condition permits.

\section{Conclusions}

The management of SVT after the development of hydrops can be challenging, to the mother as well as the medical team. Prolonged therapy for up to 2 weeks, with the use of multiple medications might be required to achieve cardioversion, provided the mother is tolerating the medications well. It becomes crucial to ensure the parents are well informed about the benefits of the treatment and the potential side effects to ensure maximum compliance. Once sinus rhythm is achieved, improvement is seen in the hydrops with significantly improved perinatal outcome.

Ethical approval: Approval was obtained from the hospital Medical Research Centre (ref MRC-04-18-090) for waiver of informed consent, provided that patient's name and other patient identifiable details would be kept anonymous and that no photograph which can identify the patient be published.

Author contributions: All the authors have accepted responsibility for the entire content of this submitted manuscript and approved submission.

Research funding: The publication of this article was funded by the Qatar National Library.

Employment or leadership: None declared.

Honorarium: None declared.

Competing interests: The funding organization(s) played no role in the study design; in the collection, analysis, and interpretation of data; in the writing of the report; or in the decision to submit the report for publication.

\section{References}

[1] van Engelen AD, Weijtens O, Brenner ]I, Kleinman CS, Copel JA, Stoutenbeek P, et al. Management outcome and follow-up of fetal tachycardia. J Am Coll Cardiol. 1994;24:1371-5.

[2] McCurdy CM, Reed KL. Fetal arrhythmias. In: Copel JA, Reed KL, editors. Doppler ultrasound in obstetrics and gynecology. New York, NY: Raven Press; 1995. p. 253.

[3] Kleinman CS, Nehgme R, Copel JA. Fetal Cardiac arrhythmias: diagnosis and therapy. In: Creasy RK, Resnik R, editors. Maternal-Fetal Medicine, 4th ed. Philadelphia, PA: WB Saunders Co; 199. p. 301.

[4] Cuneo BF, Strasburger JF. Management strategy for fetal tachycardia. Obstet Cynecol. 2000;96:575-81.

[5] Strizek B, Berg C, Cottschalk I, Herberg U, Ceipel A, Cembruch U. High-dose flecainide is the most effective treatment of fetal supraventricular tachycardia. Heart Rhythm. 2016;13:1283-8.

[6] Hill CD, Kovach ]R, Saudek DE, Singh AK, Wehrheim K, Frommelt MA. Trans placental treatment of fetal tachycardia: a systematic review and meta-analysis. Prenat Diagn. 2017;37:1076-83.

[7] Alsaied T, Baskar S, Fares M, Alahdab F, Czosek R], Murad MH, et al. First-line antiarrhythmic trans placental treatment for fetal tachyarrhythmia: a systematic review and meta-analysis. J Am Heart Assoc. 2017;6:Epub 2017.

[8] Strasburger JF, Cuneo BF, Michon MM, Gotteiner NL, Deal B], McGregor SN, et al. Amiodarone therapy for drug-refractory fetal tachycardia. Circulation. 2004;109:375-9.

[9] Oudijk MA, Michon MM, Kleinman CS, Kapusta L, Stoutenbeek P, Visser CH, et al. Sotalol in the treatment of fetal dysrhythmias. Circulation. 2000;101:2721-6. 